\title{
Combination of Solid Phase Extraction With Dispersive Liquid-liquid Microextraction for Speciation of Thallium in Water Samples by Electrothermal Vaporization ICP-MS
}

\author{
Shizhong Chen*, Dengbo Lu, and Shengping Zhu \\ College of Food Science and Engineering, Wuhan Polytechnic University, Wuhan 430023, P.R. China
}

\section{INTRODUCTION}

It is well known that thallium [Tl(I) and Tl(III)] is an element with greater toxicity to humans, plants, and animals than $\mathrm{Pb}, \mathrm{Cd}$, and $\mathrm{Hg}(1,2)$. $\mathrm{Tl}$ is mainly present as Tl(I) and Tl(III), and each oxidation is different from a toxicological point of view (3). In fact, it has been stated that Tl(III) is more toxic than Tl(I) and causes creation of reactive oxygen species (4-6). Therefore, it is necessary not only to determine total $\mathrm{Tl}$, but also to determine Tl speciation to evaluate its human health risk. The main difficulty of speciation analysis for $\mathrm{Tl}$ in environmental and biological samples lies on its low concentration level and complex matrix interferences (7). In order to solve the problems mentioned above, an effective separation and preconcentration technique for different species is usually required before their determination.

Solid phase extraction (SPE) as a classical preconcentration and separation method has received increasing attention for an element and its speciation analysis due to its simple operation, fast kinetics, rapid phase separation, and potential sample cleanup ability due to the selective adsorption of the analytes and matrices (8-10). For SPE, the type of adsorbent plays a fundamentally crucial role in the separation and preconcentration of different species. In recent years, various nanometer-size substances have been used for SPE adsorbents

\footnotetext{
*Corresponding autbor.

:E-mail: chenshizhong62@163.com

Tel: +862783956442
}

\begin{abstract}
Based on solid phase extraction (SPE) combined with dispersive liquid-liquid microextraction (DLLME), a novel method is described for speciation of thallium by electrothermal vaporization inductively coupled plasma mass spectrometry (ETV-ICP-MS). In the SPE step, titanium dioxide nanofibers were used for preconcentration of the analytes and removal of the matrix by the selective adsorption. The elution solution from the SPE was employed for further preconcentration and separation of the analytes with DLLME. The main factors affecting SPE and DLLME were examined in detail. Under optimal conditions, the detection limit of this method for Tl(III) was $0.039 \mathrm{pg} \mathrm{mL}^{-1}$ with relative standard deviations of $6.5 \%$. This technique exhibited a high enrichment factor of 400-fold. The concentration of $\mathrm{Tl}(\mathrm{I})$ was calculated by subtracting Tl(III) from total Tl. Total Tl was determined as Tl(III) after the oxidation of Tl(I) into Tl(III). This method was applied for the determination of Tl(I) and Tl(III) in water samples (industrial wastewater, urban sewage, and lake water) with satisfactory results.
\end{abstract}

owing to their excellent properties such as small size, large specific surface area, good mechanical strength, and high chemical stability (11-15). Titanium dioxide nanofibers (TDNFs) possess some unique properties such as large specific surface area, high chemical stability, corrosion resistance, nontoxicity and low cost, which makes them a promising candidate as adsorbents. However, studies on TDNFs used as a solid phase extraction adsorbent for Tl speciation have not been reported in the literature. SPE has potential sample cleanup ability, but suffers from requiring a long analysis time for large sample volumes containing very low analyte concentrations and low EF.

Since the mid-to-late 1990s, a series of liquid phase microextraction (LPME) procedures have emerged in the analytical sciences, including single-drop microextraction, hollow fiber liquid phase microextraction, solidified floating organic drop microextraction, and dispersive liquid-liquid microextraction (DLLME) (16-18). Despite the many benefits of LPME such as simplicity, low cost, rapid phase transfer and high enrichment factor (EF), its application is considerably limited because of a complex or "dirty" sample matrix (such as an organic solvent matrix). Thus, the majority of works reporting the use of LPME are focused on the analysis of elemental speciation in water samples with simple matrices (19). It should be pointed out here that each technique has its advantages and disadvantages. Recently, some investigations showed that an important strategy is the coupling of different pretreatment techniques for the determination of analytes with an extremely low concentration level in real samples (20-24). As described previously, SPE has excellent sample cleanup ability and LPME has high EF. The combination of SPE and LPME may be a potential sample pretreatment technique. 
Inductively coupled plasma mass spectrometry (ICP-MS) has been proven to be one of the most powerful analysis tools for elements and their speciation due to its merits of high sensitivity, wide linear dynamic range, and rapid multielement detection capability (25-34). However, it has low tolerance toward organic solvents, and a high percentage of organic solvent in a sample may destabilize or extinguish ICP discharge owing to relatively high solvent vapor pressure. In addition, the liquid nebulization sample introduction procedure for conventional ICP-MS is not compatible with LPME because of its large sample consumption. For these reason, the applications reporting the use of LPME for elements and their speciation have been performed by AAS in most cases (35). Electrothermal vaporization (ETV) as a sample introduction device for ICP-MS has the advantages of high introduction efficiency, low absolute detection limit, and partial or complete removal of the organic/inorganic solvent/matrix (36-38). Moreover, the microamount of sample requirement of ETV matches that of LPME well. In addition, the use of a chemical modifier in ETV, especially an organic chelating reagent, could change refractory elements to their volatile species and greatly improve the analytical performance of ETV-ICPMS (39-41).

In the present work, a method of combining SPE with DLLME was developed for Tl speciation [Tl(III) and Tl(I)] by ETV-ICP-MS. A microcolumn packed with TDNFs as the adsorbent was used for the concentration of the analytes. The elution solution from SPE was used for further preconcentration and separation of the analytes with DLLME using 1-(2-pyridylazo)-2-naphthol (PAN) as a chelating reagent and $\mathrm{CCl}_{4}$ as an extraction solvent. The extracts from DLLME were used for ETV-ICP-MS determination with
PAN as a chemical modifier. The main factors affecting SPE and DLLME were investigated in detail. This method was applied for the determination of Tl(I) and Tl(III) in water samples with satisfactory results.

\section{EXPERIMENTAL}

\section{Instrumentation}

A Series-7 ICP-MS system (Thermo Fisher Scientific Corporation, USA), equipped with a modified commercially available WF-4C graphite furnace (Beijing Second Optics, P.R. China) as an electrothermal vaporizer, was used for the determination of the analytes. The ICP-MS operating parameters were optimized with a concentric glass nebulizer prior to connection with the ETV device. A pyrolytic graphite tube was used throughout this work. The working conditions of ETV-ICP-MS are summarized in Table I. The $\mathrm{pH}$ value of the solu-

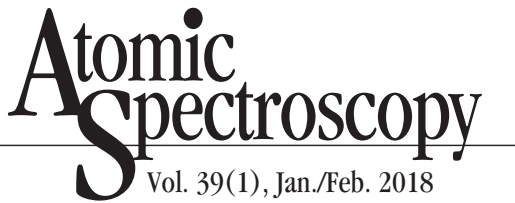

tion was controlled with a $\mathrm{pH}$ meter (Thermo Elemental Corporation, USA) supplied with a combined electrode. An Ethos T microwave digestion device (Milestone, Italy) was used for sample digestion. A KQ-50E ultrasonic bath (Kunshan Ultrasonic Instrument Co., Ltd., Suzhou, P.R. China) was used for obtaining fine droplets. A TG16-WS centrifuge (Hunan Xiangyi Centrifuge Instrument Co., Ltd., Changsha, P.R. China) was used to accelerate phase separation.

\section{Reagents and Standard Solutions}

Stock standard solutions (1.0 $\mathrm{mg} \mathrm{mL}^{-1}$ ) of Tl(I) and Tl(III) were obtained by dissolving appropriate amounts of $\mathrm{TlNO}_{3}$ and $\mathrm{Tl}\left(\mathrm{NO}_{3}\right)_{3} \cdot 3 \mathrm{H}_{2} \mathrm{O}$ (Shanghai Reagent Factory, Shanghai, P.R. China) in $1.0 \%(\mathrm{v} / \mathrm{v}) \mathrm{HNO}_{3}$ solution, respectively. Working solutions were prepared daily by appropriate dilution of the stock solutions. The PAN

TABLE I

Operating Parameters for ETV-ICP-MS

\begin{tabular}{ll}
\hline ICP-MS & \\
\hline Plasma Power & $1300 \mathrm{~W}$ \\
Plasma Argon Flow Rate & $14.5 \mathrm{~L} \mathrm{~min}^{-1}$ \\
Auxiliary Argon Flow Rate & $0.75 \mathrm{~L} \mathrm{~min}^{-1}$ \\
Nebulizer Argon Flow Rate & $0.91 \mathrm{~L} \mathrm{~min}^{-1}$ \\
Sampler Orifice (Ni) & $1.1 \mathrm{~mm}$ \\
Skimmer Orifice (Ni) & $0.7 \mathrm{~mm}$ \\
Acquisition Mode & Peak jumping \\
Number of Sweep & 100 \\
Dwell Time & $10 \mathrm{~ms}$ \\
Acquisition Time & $40 \mathrm{~s}$ \\
Number of Measurements per Peak & 3 \\
Isotope & $205 \mathrm{Tl}$ \\
\hline ETV & \\
\hline Sample Volume & $10 \mu \mathrm{L}$ \\
Carrier Gas Argon Flow Rate & $0.42 \mathrm{~L} \mathrm{~min}-1$ \\
Drying Step & $100{ }^{\circ} \mathrm{C}$, ramp10 s, hold $10 \mathrm{~s}$ \\
Ashing Step & $200{ }^{\circ} \mathrm{C}$, ramp $10 \mathrm{~s}$, hold $20 \mathrm{~s}$ \\
Vaporization Step & $2000^{\circ} \mathrm{C}$, hold $4 \mathrm{~s}$ \\
Clear-out Step & $2500{ }^{\circ} \mathrm{C}$, hold $4 \mathrm{~s}$ \\
\hline
\end{tabular}


solution was prepared by dissolving proper amounts of PAN (Shanghai Reagent Factory, P.R. China) in ethanol. Chlorobenzene, carbon tetrachloride, 1,2-dichloroethane, chloroform, acetone, ethanol, and methanol were purchased from Shanghai Reagent Factory (Shanghai, P.R. China). Unless otherwise stated, all reagents were of high purity or at least of analytical grade. High purity deionized water obtained from a Milli-Q ${ }^{\circledR}$ A10 system (Millipore Corporation, USA) was used throughout this work. All glass and polypropylene wares were kept in $3.0 \mathrm{~mol} \mathrm{~L}^{-1} \mathrm{HNO}_{3}$ solution for at least one night, then rinsed with $0.1 \mathrm{~mol} \mathrm{~L}^{-1} \mathrm{HNO}_{3}$ solution, and subsequently with deionized water before use. TDNFs were synthesized by facile electrospinning and a subsequent annealing method in our laboratory. Their diameter is mainly in the range of 350-450 $\mathrm{nm}$.

\section{Extraction Procedures}

\section{Solid Phase Extraction}

A PTFE microcolumn $(20 \mathrm{~mm} \times$ $3.0 \mathrm{~mm}$ i.d.) plugged with a small portion of glass wool at both ends was filled with $60 \mathrm{mg}$ of TDNFs. Before use, $5.0 \mathrm{~mL}$ of $1.0 \mathrm{~mol} \mathrm{~L}^{-1}$ $\mathrm{HNO}_{3}$ solution and $10 \mathrm{~mL}$ of deionized water were passed through the column to clean and condition it, respectively. Then, an aliquot of a 20-mL sample solution was passed through the column by using a peristaltic pump. The retained analytes were eluted with $4.0 \mathrm{~mL}$ of 0.6 mol L-1 $\mathrm{HNO}_{3}$ solution. Finally, $4.0 \mathrm{~mL}$ of desorption solution was transferred into a $10-\mathrm{mL}$ vial for DLLME.

\section{Dispersive Liquid-liquid Microextraction}

Aliquots of a 4.0-mL sample solution containing the analytes and $8.0 \times 10^{-3} \mathrm{~mol} \mathrm{~L}^{-1}$ of PAN were adjusted to $\mathrm{pH} 8.0$ and placed in a 10-mL screw cap test tube with a conic bottom. A $0.5-\mathrm{mL}$ amount of ethanol (as dispersive solvent) containing $50 \mu \mathrm{L}$ of $\mathrm{CCl}_{4}$ (as extraction solvent) was rapidly injected into the sample solution by using a $100-\mu \mathrm{L}$ syringe. A cloudy solution was formed immediately in the test tube, and the Tl-PAN complex was extracted into the fine droplets of $\mathrm{CCl}_{4}$. Then, the mixture was centrifuged for 3 minutes at $5000 \mathrm{rpm}$. After that, a small droplet of $\mathrm{CCl}_{4}$ (approximately $45 \mu \mathrm{L}$ ) was added to settle at the bottom of the conical test tube. Finally, $10 \mu \mathrm{L}$ of the sedimented phase was withdrawn with a micro-syringe and injected into the ETV for ICP-MS measurement.

\section{ETV-ICP-MS Procedure}

After the ETV unit had been connected to the ICP-MS, $10 \mu \mathrm{L}$ of the analytes was injected into the graphite furnace. During the drying and charring process, the dosing hole of the graphite furnace was kept open to remove the water and the organic vapor. Then, it was sealed with a graphite probe within 5-8 seconds prior to the vaporization step. The vaporized analytes were swept into the plasma excitation source by a carrier gas, and the peak-hop transient mode for data acquisition was used for the determination of the analytes.

\section{RESULTS AND DISCUSSION}

\section{Selection of SPE Conditions}

\section{Effect of Sample Solution $p H$}

The $\mathrm{pH}$ value plays an important role in the adsorption of the analytes on sorbents. Thus, the effect of sample $\mathrm{pH}$ on the adsorption of the analytes on TDNFs was investigated in the $\mathrm{pH}$ range of 1.0-8.0. The results in Figure 1 reveal that in the $\mathrm{pH}$ range of 5.0-8.0, Tl(III) and $\mathrm{Tl}(\mathrm{I})$ were quantitatively retained on the TDNFs. Thus, $\mathrm{pH}$ 6.0 was selected for the preconcentration of the sum of Tl(III) and Tl(I) by SPE in the following experiments. In addition, the adsorption capacity was investigated by the method recommended in the literature (42). The adsorption capacities of TDNFs for $\mathrm{Tl}(\mathrm{I})$ and $\mathrm{Tl}(\mathrm{III})$ are 3.8 and $4.5 \mathrm{mg} \mathrm{g}^{-1}$, respectively.

\section{Effect of Elution Parameter}

From Figure 1, it can be seen that the adsorption percentages of the analytes on the TDNFs decreased significantly at $\mathrm{pH}<1.0$. Therefore, the different concentrations of $\mathrm{HNO}_{3}$ solution were studied for the desorption of the retained analytes. The experimental results showed that the retained species could be eluted quantitatively with 0.60 mol L-1 $\mathrm{HNO}_{3}$ solution. Furthermore, the effect of eluent volume on the recoveries of the analytes was also studied with $0.60 \mathrm{~mol} \mathrm{~L}^{-1} \mathrm{HNO}_{3}$ solution. The experimental results indicated that quantitative recoveries $(>90.0 \%)$ could be obtained with $4.0 \mathrm{~mL}$ of $0.60 \mathrm{~mol} \mathrm{~L}^{-1} \mathrm{HNO}_{3}$ solution.

In addition, the effect of eluent flow rate on the recovery of the analytes was examined by keeping $4.0 \mathrm{~mL}$ eluent volume and 0.60 mol L-1 $\mathrm{HNO}_{3}$ solution. The experimental results indicated that any flow rate between 0.2 and 1.0 $\mathrm{mL} \mathrm{min}^{-1}$ can be used for the quantitative elution of the analytes. To obtain a short analysis time, the flow rate of the eluent was set at $1.0 \mathrm{~mL} \mathrm{~min}^{-1}$ for this work.

\section{Effect of Sample Flow Rate and Volume}

The flow rate of the sample solution was optimized in the range of 0.1 to $2.5 \mathrm{~mL} \mathrm{~min}^{-1}$. The experimental results indicated that there was a decrease in the recoveries of the analytes at a flow rate higher than $1.6 \mathrm{~mL} \mathrm{~min}^{-1}$. Therefore, all subsequent experiments were performed

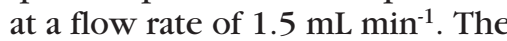
effect of sample volume on the adsorption of the analytes was studied in the range of 10-200 mL containing $1.0 \mathrm{ng}$ of the analytes. The experimental results showed that 
quantitative recoveries (> 90\%) were obtained for sample volumes up to $120 \mathrm{~mL}$. Considering the analysis time, a 20-mL sample volume was used for the analysis of real samples.

\section{Optimization of DLLME Factors}

\section{Effect of Sample $p H$}

Due to its influence on the existing form of the analytes and PAN, the sample $\mathrm{pH}$ is a key factor in the separation and concentration of the analytes, and affects the Tl-PAN complex formation with necessary hydrophobicity and the subsequent extraction. Thus, the effect of sample $\mathrm{pH}$ on the signal intensity of the analytes was investigated in detail. The results are shown in Figure 2. As can be seen, maximum signal intensity of Tl(III) was obtained in the $\mathrm{pH}$ range of 7.0-10.0, whereas the signal of $\mathrm{Tl}(\mathrm{I})$ was not recorded in the same $\mathrm{pH}$ range. The reason for the fact mentioned above is that at the corresponding $\mathrm{pH}$ range, $\mathrm{Tl}(\mathrm{I})$ cannot form a stable and hydrophobic complex with PAN. Based on these results, a $\mathrm{pH}$ of 8.0 was used for the separation of Tl(III) and T(I). It should be pointed out that the concentration of Tl(I) was calculated by subtract- ing Tl(III) from total Tl. The total $\mathrm{Tl}$ was determined as Tl(III) after the oxidation of $\mathrm{Tl}$ (I) into Tl(III) as reported in the literature (43).

\section{Effect of Extraction Solvent and Its Volume}

In general, the extraction solvent should have low solubility in water and excellent extraction ability for the target analytes. In addition, it should have higher density than water to make the phase separation easy. For this purpose, chlorobenzene, carbon tetrachloride, 1,2dichloroethane, and chloroform were investigated as the extraction solvents. The experimental results show that carbon tetrachloride $\left(\mathrm{CCl}_{4}\right)$ was found to have the best extraction efficiency among the tested solvents. Thus, $\mathrm{CCl}_{4}$ was selected as the optimal extraction solvent.

The effect of extraction solvent volume on the extraction efficiency was also investigated in the range of $10-60 \mu \mathrm{L}$. The experimental results illustrate that the signal intensity of the analytes kept almost constant when the extraction solvent volume varied from 10 to 50 $\mu \mathrm{L}$. However, the signal intensity

\section{Atomic Apectroscopy \\ $\bigcup$ Vol. 39(1), Jan./Feb. 2018}

of the analytes decreased by further increasing the volume above $50 \mu \mathrm{L}$. Therefore, $50 \mu \mathrm{L}$ of $\mathrm{CCl}_{4}$ was used in this work.

\section{Effect of Type and Volume of Dispersive Solvent}

In order to form a stable emulsion, the dispersive solvent in DLLME should be miscible with both water and the extraction solvent. For this purpose, acetone, ethanol, and methanol were studied as the dispersive solvents. The experimental results show that the recoveries for acetone, ethanol, and methanol were $95.8 \%, 97.2 \%$, and $98.1 \%$, respectively. The highest recovery was obtained using methanol, but ethanol has low toxicity and is low cost. Therefore, it was selected as the dispersing solvent.

In addition, the effect of the volume of ethanol on the signal intensity of the analytes was investigated in the range of $0.1-1.0 \mathrm{~mL}$. The experimental results show that the signal intensity for the analytes was increased with an increase in the volume of ethanol from 0.2 to 0.5 $\mathrm{mL}$, and decreased when the volume of ethanol exceeded $0.5 \mathrm{~mL}$.

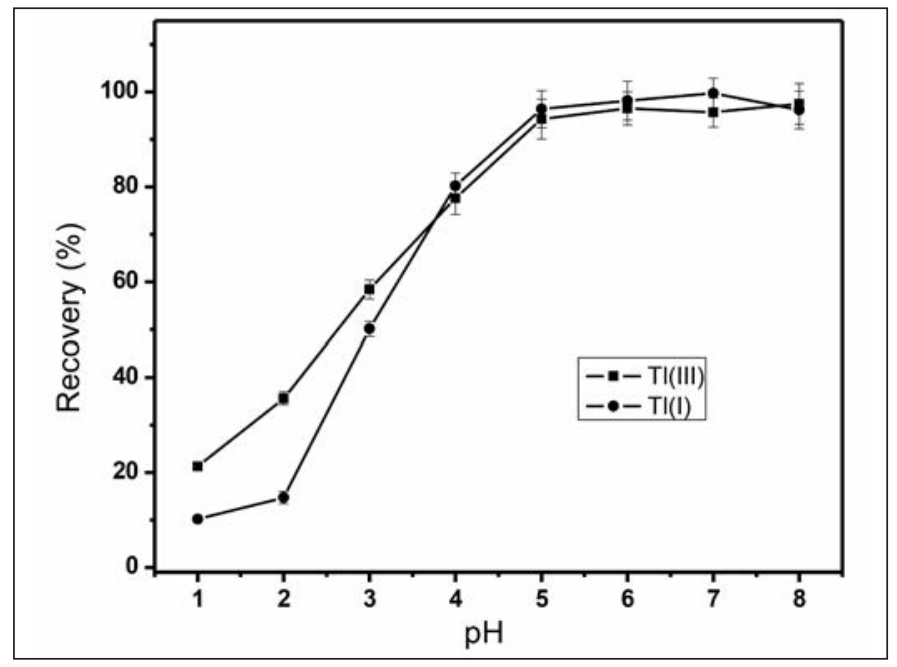

Fig. 1. Effect of pH on the adsorption of analytes on TDNFs in SPE. Tl(III) and Tl(I): $1.0 \mathrm{ng} \mathrm{mL}^{-1}$; sample volume: $20 \mathrm{~mL}$.

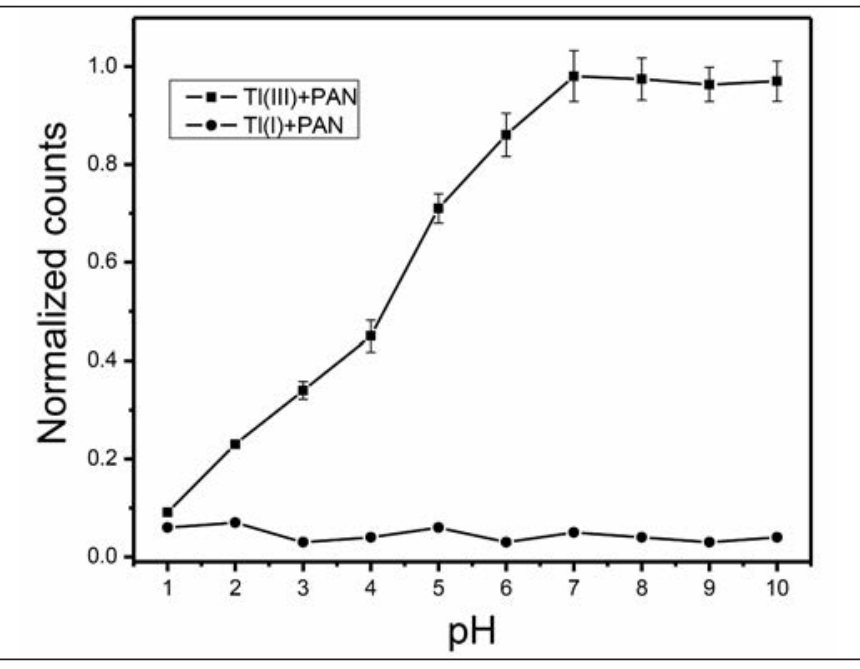

Fig. 2. Effect of sample pH on signal intensity of analytes in DLLME. Tl(III) and Tl(I): $1.0 \mathrm{ng} \mathrm{mL}^{-1}$; vaporization temperature: $2000^{\circ} \mathrm{C}$. 
Therefore, $0.5 \mathrm{~mL}$ of ethanol was selected as the optimum volume to achieve a stable cloudy solution.

\section{Influence of PAN Amount}

The influence of PAN concentration on the signal intensity of the analytes was studied in the concentration range of $0.5 \times 10^{-3}-2.0 \times 10^{-2}$ $\mathrm{mol} \mathrm{L}^{-1}$. The experimental results indicate that the signal intensities of the analytes were increased with an increase in PAN concentration from $0.5 \times 10^{-3}$ to $5.0 \times 10^{-3} \mathrm{~mol} \mathrm{~L}^{-1}$, and then kept nearly constant when the concentration of PAN exceeded $5.0 \times 10^{-3} \mathrm{~mol} \mathrm{~L}^{-1}$. Thus, a relatively high concentration of $8.0 \times 10^{-3} \mathrm{~mol} \mathrm{~L}^{-1}$ PAN was selected for all further experiments to account for the other extractable species that potentially interfere with the assaying of Tl speciation.

\section{Effect of Extraction Time}

The effect of extraction time was evaluated in the range of 5 seconds to 2 minutes. The experimental results show that the extraction time has no significant effect on extraction efficiency, indicating an extremely large surface area between the extraction solvent and the aqueous phase and a fast extraction dynamics. Hence, the extraction time can be kept very short, which is the remarkable advantage of DLLME. Consequently, 5 seconds was selected for extraction of the analytes.

\section{Effect of Potential Interfering Ions}

In order to evaluate the application potential of this method, the effect of various cations and anions commonly existing in natural water samples on the extraction and determination of analytes was examined. The tolerance limit of coexisting ion is defined as the largest amount making the variation of less than $10 \%$ in the recovery of the analytes. The results show that $2000 \mathrm{mg} \mathrm{L}^{-1} \mathrm{Cl}^{-}$and $\mathrm{NO}_{3}{ }^{-}, 1000$ $\mathrm{mg} \mathrm{L}^{-1} \mathrm{SO}_{4}^{2-}, \mathrm{SiO}_{3}{ }^{2-}$ and $\mathrm{PO}_{4}^{3-}$,
$2000 \mathrm{mg} \mathrm{L}^{-1} \mathrm{~K}^{+}$and $\mathrm{Na}^{+}, 1000 \mathrm{mg} \mathrm{L}^{-1}$ $\mathrm{Ca}^{2+}$ and $\mathrm{Mg}^{2+}, 3.0 \mathrm{mg} \mathrm{L}^{-1} \mathrm{Al}^{3+}$ and $\mathrm{Fe}^{3+}$ had no significant effect on the extraction and determination of the analytes.

\section{Optimization of ETV Conditions}

In order to obtain good sensitivity and accuracy, the ETV temperature program was optimized and selected for this work. A drying temperature of $100^{\circ} \mathrm{C}$ and a drying time of 10 seconds were used for removal of the water vapor during the drying step; a pyrolysis temperature of $200{ }^{\circ} \mathrm{C}$ and a pyrolysis time of 20 seconds were used for in situ removal of the extraction solvent from the furnace without signal loss of the analytes. In addition, the effect of vaporization temperature on the signal intensity of the analytes was studied with a vaporization time of 4 seconds. The experimental results showed that with PAN as the chemical modifier, a weak signal for the analytes appeared at $500{ }^{\circ} \mathrm{C}$, and the signal increased gradually with an increase in vaporization temperature. Maximum signal was obtained at $1800^{\circ} \mathrm{C}$ for the analytes, and then kept constant with a further increase of the vaporization temperature to $2200{ }^{\circ} \mathrm{C}$. Thus, a vaporization temperature of $2000{ }^{\circ} \mathrm{C}$ with a vaporization time of 4 seconds was used in this work.

\section{Analytical Performances}

Under the optimized conditions, some important parameters of this method, including precision, linear range of the calibration curve, and detection limits, were validated. The detection limit of TI(III), based on three times the standard deviation of the blank solution, was $0.039 \mathrm{pg} \mathrm{mL}^{-1}$ with a 400 -fold enrichment factor (EF), (EF, defined as the slope ratio of the calibration curve obtained by ETV-ICP-MS with and without SPE-DLLME pretreatment). The linear dynamic range of the calibration curve covered three orders of magnitude with a correla- tion coefficient better than 0.9967 . The relative standard deviation was $6.5 \%$ for $\mathrm{Tl}(\mathrm{III})$ ( $\mathrm{c}=0.1 \mathrm{ng} \mathrm{mL}^{-1}$, $\mathrm{n}=9$ ).

\section{Sample Analysis}

Water samples (Wuhan, P.R. China), including industrial wastewater, urban sewage, and lake water, were collected in 50-mL polyethylene containers. They were filtered through a $0.45-\mu \mathrm{m}$ membrane filter and then stored in polyethylene bottles at $4^{\circ} \mathrm{C}$ for future use. This method was applied for the determination of Tl(I) and Tl(III) in the water samples mentioned above. The results are listed in Table II. The recoveries of the analytes were in the range of 92.0-102\%.

\section{CONCLUSION}

In this work, the combination of SPE with DLLME was developed for the determination of $\mathrm{Tl}(\mathrm{I})$ and Tl(III) in water samples prior to ETV-ICP-MS. A microcolumn packed with TDNFs as the adsorbent was used for the concentration of the analytes and the removal of the matrices, including the inorganic or organic matrices in the analyzed water samples. The elution solution from SPE was used for further preconcentration and separation of the analytes with DLLME, using PAN as the chelating reagent and $\mathrm{CCl}_{4}$ as an extraction solvent. The extracts from DLLME were used for ETV-ICP-MS determination with PAN as the chemical modifier. This method has the features of high EF (400-fold), low detection limit, and good accuracy. The combination of different preconcentration/separation methods not only has excellent sample cleanup ability, but also improves the analytical performance. It is likely to become a potential technique for $\mathrm{Tl}$ speciation in real samples. 


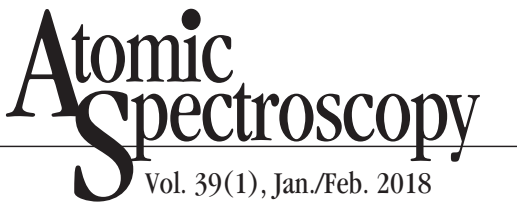

TABLE II

Analytical Results of Water Samples

\begin{tabular}{|c|c|c|c|c|c|c|c|}
\hline \multirow[t]{2}{*}{ Sample } & \multicolumn{2}{|c|}{ Added (ng mL $\left.\mathrm{mL}^{-1}\right)$} & \multicolumn{3}{|c|}{ Found $^{\mathrm{a}}\left(\mathrm{ng} \mathrm{mL}^{-1}\right)$} & \multicolumn{2}{|c|}{ Recovery (\%) } \\
\hline & $\mathrm{Tl}(\mathrm{I})$ & Tl(III) & $\mathrm{Tl}(\mathrm{I})$ & Tl(III) & Total & $\mathrm{Tl}(\mathrm{I})$ & Tl(III) \\
\hline \multicolumn{8}{|l|}{ Industrial Wastewater } \\
\hline & 0 & 0 & $6.23 \pm 0.34$ & $2.11 \pm 0.15$ & $8.34 \pm 0.42$ & - & - \\
\hline & 3.0 & 3.0 & $9.05 \pm 0.57$ & $5.02 \pm 0.31$ & $14.07 \pm 0.62$ & 94.0 & 97.0 \\
\hline & 5.0 & 5.0 & $10.99 \pm 0.45$ & $6.86 \pm 0.32$ & $17.85 \pm 0.91$ & 95.2 & 95.0 \\
\hline \multirow[t]{3}{*}{ Urban Sewage } & 0 & 0 & $1.56 \pm 0.09$ & $0.72 \pm 0.05$ & $2.28 \pm 0.13$ & - & - \\
\hline & 1.0 & 1.0 & $2.54 \pm 0.15$ & $1.64 \pm 0.12$ & $4.18 \pm 0.27$ & 98.0 & 92.0 \\
\hline & 2.0 & 2.0 & $3.53 \pm 0.17$ & $2.74 \pm 0.15$ & $6.27 \pm 0.29$ & 98.5 & 101 \\
\hline \multirow[t]{3}{*}{ Dongxi Lake Water } & 0 & 0 & $0.41 \pm 0.02$ & $0.12 \pm 0.06$ & $0.53 \pm 0.03$ & - & - \\
\hline & 0.5 & 0.5 & $0.89 \pm 0.05$ & $0.63 \pm 0.04$ & $1.52 \pm 0.10$ & 96.0 & 102 \\
\hline & 1.0 & 1.0 & $1.38 \pm 0.09$ & $1.05 \pm 0.07$ & $2.43 \pm 0.12$ & 97.0 & 93.0 \\
\hline
\end{tabular}

${ }^{a}$ Mean value \pm standard deviation, $n=5$.

\section{ACKNOWLEDGMENTS}

The authors are grateful for the financial support of the Special Fund for Agroscientific Research in the Public Interest (Project No. 201503135-22)

Received June 10, 2017.

\section{REFERENCES}

1. G. Kazantzis, Environ. Geochem. Health 22, 275 (2000).

2. A. Sasmaz, O. Sen , G. Kaya, M. Yaman, and A. Sagiroglu, At. Spectrosc. 28(5), 157 (2007).

3. Z. Xu, S. Xu, and Z. Fang, At. Spectrosc. 21(1) 17, (2000).

4. R.A. Gil, P.H. Pacheco, P. Smichowski, R.A. Olsina, and L.D. Martinez, Microchim. Acta 167(34), 187 (2009).

5. C. Lan and T. Lin, Ecotoxic. Environ. Saf. 61(3), 432 (2005).

6. P. Jalal, E.M. Reza, and D. Bahram, Environ. Toxicol. 25(5), 456 (2010).

7. X. Guo, M. He, B. Chen, and B. Hu, Talanta 94, 70 (2012).

8. J. Yan, S. Chen, J. Li, Y. He, and D. Lu, At. Spectrosc. 38(2), 42 (2017).

9. H. Fazelirad and M.A. Taher,
Microchim. Acta 181(5-6), 655 (2014).

10. S. Sivrikaya, M. Imamoglu, and D. Kara, At. Spectrosc. 35(4), 168 (2014).

11. D. Xiao, T. Lu, R. Zeng, and Y. Bi, Microchim. Acta 183(10), 2655 (2016).

12. Y. He, S. Chen, X. Zhou, and X. Wang, At. Spectrosc. 33(4), 117 (2012).

13. S. Chen, L. Zhu, D. Lu, X. Cheng, and $\mathrm{X}$. Zhou, Microchim. Acta 169(1-2), 123 (2010).

14. S. Chen, S. Zhu, Y. He, and D. Lu, Food Chem. 150, 254 (2014).

15. S. Chen, J. Li, Y. He, and D. Lu, At. Spectrosc. 37(3), 96 (2016).

16. M.A. Farajzadeh, S.M. Sorouraddin, and R.A. Mohammad, Microchim. Acta 181(9-10), 829 (2014).

17. P. Francisco, L. Isela, and B. Carlos, Anal. Chim. Acta 669 (1-2), 1 (2010).

18. S. Chen, Y. He, S. Zhu, and D. Lu, At. Spectrosc. 36(6), 247 (2015).

19. H. Bin, H. Man, C. Beibei, and X. Linbo, Spectrochim. Acta Part B 86, 14 (2013).

20. D. Djavanshir, A.F. Mir, M.S. Saeed, and B. Tahmineh, J Chromatogr. A, 1248, 24 (2012).

21. Y. Yamini, M. Faraji, and M. Adeli, Microchim. Acta 182(7-8), 1491
(2015).

22. C. Diao, C. Li, X. Yang, A. Sun, and R. Liu, Microchim. Acta 183(3), 1261 (2016).

23. L. Thabiso and J.G. Mosotho, Anal. Chem. Res. 10, 28 (2016).

24. S. Chen, J. Yan, J. Li, Y. Zhang, and D. Lu, At. Spectrosc. 38(1), 12 (2017).

25. J. Moreda-Pineiro, C. Garcia-Sartal, C. Barciela-Alonso, P. BarcielaAlonso, P. Lopez-Mahia, S. Muniategui-Lorenzo, D. Prada-Rodriguez, and P. BermejoBarrera, At. Spectrosc. 37(6), 218 (2016).

26. V. Padmasubashini and G. Chakrapani, At. Spectrosc. 37(6), 229 (2016).

27. J. Zhang, Y. Dong, and Z. Xu, At. Spectrosc. 37(4), 131 (2016).

28. S.A. Ozen and M. Yaman, At. Spectrosc. 37(4), 136 (2016).

29. M.C. Barciela-Alonso, M.C. Otero, M.R.D. Gonzalez, E. Pena-Vazquez, and P. Bermejo-Barrera, At. Spectrosc. 37(3), 91 (2016).

30. S. Chen, J. Li, Y. He, and D. Lu, At. Spectrosc. 37(3), 96 (2016).

31. J. Zhang, X. Wang, Y. Dong, Z. Xu, and G. Li, At. Spectrosc. 37(1), 1 (2016).

32. W. Guo, Y. Wang, J. Li, Y. Peng, L. Jin, Q. Guo, and S. Hu, At. Spectrosc. 37(1), 7 (2016). 
33. S. Chen, Y. He, Y. Zhang, and D. Lu, At. Spectrosc. 36(4), 153 (2015).

34. S. Chen, S. Zhu, and D. Lu, At. Spectrosc. 36(5), 196 (2015).

35. C. Inmaculada, P. Francisco, L. Isela, and B. Carlos, Anal. Chim. Acta 936, 12 (2016).

36. S. Chen, J. Li, D. Lu, and S. Zhu, At. Spectrosc. 37(1), 13 (2016).

37. D.L.G. Borges, B. Welz, and A.J. Curtius, Microchim. Acta 159(1-2), 19 (2007)

38. Z. Ying, M. Xuefei, L. Jixin, W. Min, Q. Yongzhong, G. Chengling, and Q. Yuehan, Spectrochim. Acta Part B 118, 119 (2016)

39. L. Ying, H. Man, C. Beibei, and $\mathrm{H}$. Bin, Talanta 142, 213 (2015).

40. S. Chen, S. Zhu, and D. Lu, At. Spectrosc. 36(3), 128 (2015).

41. W. Hsu, S. Jiang, and A.C. Sahayam, Talanta 17, 268 (2013).

42. A. Maqieira, H. A. M. Elmahadi, and R. Puchades, Anal. Chem. 66, 3632 (1994).

43. A.I. Vogel, A Text Book of Quantitative Inorganic Analysis, 6th ed. Longman, London, UK, p. 159 (2000). 